\title{
Die Wiederentdeckung von Byzanz: Die kretische Ikone von Göttingen und die Koimesis-Darstellung in der byzantinischen und postbyzantinischen Epoche
}

Mit 10 Abb. auf Tafel I-VII

\begin{abstract}
What are the similarities and the differences of icons from the same workshop depicting the same subject? An important portable icon with the representation of the Dormition of the Virgin, hitherto unknown, preserved today in the Art Collection of the University of Göttingen, helps answering this question. The study deals with the fascinating journey of this icon from Venetian-dominated Crete in the 15th century to Germany of the 18th century. Furthermore, this paper shows that the icon of Göttingen belongs to a group of a numerous icons that they all derive from the same icon-workshop of the renowned Cretan painters Andreas and Nikolaos Ritzos in Candia. Finally, it turned out that this icon was also the inspiration for Cretan painters of the 17th CE such as Emmanuel Lambardos and Viktor.
\end{abstract}

Adresse: Dr. Markos Giannoulis, Institut für Byzantinistik, Byzantinische Kunstgeschichte und Neogräzistik, Geschwister-Scholl-Platz 1, 80539 München, DeUtSCHLAND; markos.giannoulis@Imu.de

Goethes scharfsinnigem Blick ist Byzanz mit seiner künstlerischen Ausprägung nicht entgangen; im Abendland war er in dieser Zeit einer der wenigen, gewiss der

Grundüberlegungen zu diesem Thema habe ich im Mai 2018 auf der 38. Tagung der ChAE in Athen

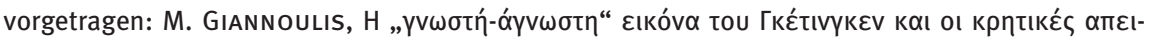

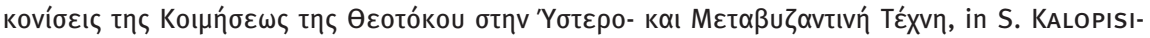
VERTI (Hrsg.), Abstracts of 38th Symposium on Byzantine and post-Byzantine Archaeology and Art (Athen 11 - 13 May 2018). Athen 2018, 47. An erster Stelle möchte ich meinen herzlichen Dank an Prof. Dr. Achim Arbeiter aussprechen, der mir den Hinweis auf die Existenz dieser Ikone gab. Dem zuvorkommenden Engagement der Kollegin Lisa Marie Roemer von der Kunstsammlung der Georg-August-Universität Göttingen ist es geschuldet, dass mir die Ikone stets uneingeschränkt zugänglich war sowie hochwertige Abbildungsvorlagen zur Verfügung standen. 
berühmteste und bedeutendste Westeuropäer, der ein besonderes und sogar ein gewissermaßen kunstwissenschaftliches Interesse an der Ikonenmalerei der Ostkirche zeigte. Wie aus seinem berühmten „Wunschzettel“1 an das russische Innenministerium hervorgeht, versucht er mehr über die byzantinischen Vorlagen der „russischen Heiligenbilder“ zu erfahren, denn - wie er selbst sagt - „ein aus den ältesten Zeiten von Konstantinopel her abgeleiteter Kunstzweig [hat] sich bis auf unsere Tage unverändert durch eine stetige Nachahmung erhalten“. ${ }^{2}$

Ein Zeitgenosse und Bekannter Goethes, Christoph Gottlieb von Murr, wandte der damals üblicherweise als „neugriechisch“ bezeichneten Tafelmalerei ebenfalls seine Aufmerksamkeit zu und erwarb eine solche Ikone. Diese Ikone, der Hauptgegenstand des vorliegenden Aufsatzes, ist bisher weder der breiteren Öffentlichkeit noch der Wissenschaft bekannt gewesen und hat daher kaum Würdigung erfahren, die ihrer kunstgeschichtlichen Bedeutung angemessen wäre. Gerade die Tatsache, dass diese Ikone in Deutschland im 18. Jh. auftaucht, macht den Fall besonders interessant, war doch die Ikonenmalerei in jener Zeit auch in Deutschland terra incognita. Wie noch zu zeigen ist, handelt es sich bei der Ikone jedoch nicht nur um ein bedeutendes Bildwerk der sogenannten kretischen Malerei, sondern auch um eines, das einer der wichtigsten Ikonen-Werkstatt von Candia, dem heutigen Heraklion auf Kreta, zugeordnet werden kann. Ihre kunstgeschichtliche Bedeutung steigt zudem aufgrund der Tatsache, dass bekannte postbyzantinische Maler anscheinend genau diese Ikone später als Vorbild für ihre eigenen Werke verwenden.

Zunächst aber einige technische Angaben: Die Ikone wird heute in der Kunstsammlung der Universität Göttingen unter der Inv.-Nr. GG 198 aufbewahrt (Abb. 1). Die recht- bzw. hochformatige Bildtafel mit den Maßen 38,5 cm $\times 31,5 \mathrm{~cm}$ und $2,5 \mathrm{~cm}(\mathrm{H} \times \mathrm{B} \times \mathrm{T})$ ist aus einem einzigen Stück Nussholz ohne Rahmen und ohne vertieftes Bildfeld angefertigt. Die alte Weisheit, dass das Holz des Nussbaums eine ausgezeichnete Wahl für die Tafelmalerei sei, weil es als Hartholz kaum von Tieren und Mikroorganismen befallen werde, lässt sich am Beispiel der Göttinger Ikone noch einmal bestätigen. Die Festigkeit der Holztafel ist ferner auf der gehobelten Rückseite mit zwei Querleisten verstärkt; die beiden wohl originalen

$1 \mathrm{Zu}$ Goethes Beziehung zu Byzanz sowie zum sog. Wunschzettel von 1814 an die Zarin-Mutter Maria Fjodorowna bzw. an das russische Innenministerium: A. EFFENBERGER, Goethe und die „russischen Heiligenbilder“. Anfänge deutscher Kunstgeschichte in Deutschland. Mainz 1990; und A. EfFenberger, Frühes Ikonenstudium in Deutschland, in B. Borkopp u. a. (Hrsg.), Studien zur Byzantinischen Kunstgeschichte. Festschrift für Horst Hallensleben zum 65. Geburtstag. Amsterdam 1995, 249-253 auch mit weiterführender Literatur.

2 Zitiert nach: EFFENBERGER, Ikonenstudium (wie oben Fußnote 1) 250. 
Querleisten aus dem gleichen Holz sind angeleimt und zusätzlich mit je drei Nägeln befestigt. Trotz dieser Maßnahmen konnte die Bildung von kleineren Rissen in der Holztafel im Laufe der Jahrhunderte nicht vermieden werden; diese wurden aber bei der letzten Restaurierung 31972 fachgerecht gekittet und retuschiert. Temperamalerei und Vergoldung auf der Vorderseite sind in sehr gutem Zustand, auch wenn typische altersbedingte Gebrauchsspuren in Form von mehreren kleinen Kratzern, Abplatzungen und Fehlstellen über die gesamte Oberfläche verteilt nicht fehlen. Durch diese Fehlstellen lässt sich feststellen, dass die relativ dicke weiße Grundierung direkt auf die Nussholz-Tafel, d.h. ohne Leinwand aufgebracht worden ist. Die Stelle mit dem größten Malschichtverlust von etwa $1 \mathrm{~cm}^{2}$ befand sich unten auf dem rechten Bildrand; sie wurde ebenfalls 1972 restauriert.

\section{Ein evangelischer Gelehrter und seine Ikone: zur Provenienz der Ikone und den ersten Schritten der modernen Ikonenrezeption in Deutschland}

Bevor wir auf die tatsächliche kunstgeschichtliche Bedeutung dieses Bildwerks eingehen, soll im Folgenden kurz seine Provenienz nachgezeichnet werden. Man fragt sich mit Sicherheit, wie eine solche Ikone den Weg in die Kunstsammlung der Göttinger Universität gefunden hat und aus welchen historischen und gesellschaftlichen Kontexten sie stammt. Die frühesten Spuren, die sich bezüglich ihrer Herkunft ermitteln lassen, führen in das Nürnberg des ausgehenden 18. Jh.s. Im Jahre 1795 hören wir zum ersten Mal von diesem Tafelbild, als es durch Kauf von der Akademie zu Nürnberg in die Kunstsammlung der damals relativ jungen Georg-August-Universität in Göttingen gelangte. ${ }^{4}$ Dieser Ankauf kam durch das Akademiemitglied Christoph Gottlieb von Murr zustande; der bekannte protestantische Nürnberger Universalgelehrte war der Vermittler und der Vorbesitzer der Ikone. Wie die Ikone in seinen Besitz gelangte, lässt sich heute nicht mehr mit Sicherheit zurückverfolgen.

3 Die Restaurierung von 1972 wurde durch Kurt Mannig ausgeführt. Der Restaurierungsbericht wird in der Kunstsammlung der Universität Göttingen unter der Akten-Nummer GG 198 aufbewahrt. Die Verbesserungen wurden von den Restautoren in etwas dunklerer Farbe durchgeführt, sodass sie bei genauerer Betrachtung sofort sichtbar sind.

4 J.D. FioRILlo, Beschreibung der Gemählde-Sammlung der Universität zu Göttingen. Göttingen 1805, 65-66. Kat. Nr. 10. 
Betrachtet man Murrs Biographie genauer, ergeben sich doch einige relevante Details: Christoph Gottlieb von Murr, ${ }^{5} 1733$ geboren, machte zunächst Karriere in der Stadtverwaltung als mercaturae vectigabilus praefectus, also etwa als Zolloder Waagamtmann. Sein Amt lastete ihn anscheinend nicht aus, da er sein Leben und seine ganze Energie eifrig den Wissenschaften widmete. Durch seine umfangreiche Tätigkeit sowohl als Herausgeber wie auch als Autor von etwa zweihundert Titeln erlangte er über die Grenzen der Stadt Nürnberg hinaus große Reputation. Das beweist zum einen sein Briefwechsel ${ }^{6}$ mit vielen bekannten Gelehrten seiner Zeit und zum anderen, dass er als Mitglied in verschiedene wissenschaftliche Gesellschaften aufgenommen wurde, darunter auch die Historische Gesellschaft in Göttingen; zum Göttinger Gelehrtenkreis stand er in enger freundschaftlicher Verbindung, was sich an seinem Beitrag zu der Bereicherung der damals neuen, gerade entstehenden Universitätssammlung zeigt. Man sollte dabei anmerken, dass für die damaligen Verhältnisse allein die Idee einer Universitätssammlung und somit die Möglichkeit eines objektnahen Forschens ein besonders innovativer Einfall war. Dass dazu byzantinische Ikonen als Anschauungsmaterial im kunstgeschichtlichen Unterricht gehören sollten, zeigt deutlich, dass schon in jener Zeit manche Menschen über breitere und tiefere Kenntnisse der Wurzeln des Faches Kunstgeschichte verfügten. Es ist durchaus möglich, dass Murr die gleiche Begeisterung für die byzantinische Kunst wie Goethe hatte. Goethe ${ }^{7}$ stand in Verbindung mit Murr, den er sogar zwei Mal auf dem Weg nach und von Italien in Nürnberg besuchte. Durch die Beziehung zu Goethe erfahren wir fernerhin, dass Murr über eine private Kunstsammlung verfügte, und dass er wie jeder gute Sammler an An- und Verkäufen ${ }^{8}$ interessiert war. Es ist denkbar, dass die Ikone durch seine Kunsthandelsaktivitäten oder seine Tätigkeit als Zollamtmann in seine Hände gelangte.

5 Zu Murrs Biographie: E. Mummenhoff, Murr, Christoph Gottlieb von, in Allgemeine Deutsche Biographie 23 (1886), 76-80; auch online verfügbar (letzter Aufruf 28.05.2019) URL: https:// www.deutsche-biographie.de/pnd11906362X.html\#adbcontent; und A. НосH, Christoph Gottlieb von Murr, in Ch. von Imhoff (Hrsg.), Berühmte Nürnberger aus neun Jahrhunderten. Nürnberg 1984, $225-227$.

6 Einen Eindruck der Bekanntschaften Murrs bekommt man durch seine Korrespondenz, die heute im „Murriana“-Nachlass in der Bayerischen Staatsbibliothek in München aufbewahrt wird. 7 Zu Goethes Bekanntschaft mit Murr: J. GRAVE, Der „ideale Kunstkörper“ Johann Wolfgang Goethe als Sammler von Graphiken und Zeichnungen. Göttingen 2006, 112-113 mit weiterführender Literatur.

8 Ebd. 122: Zu seinen Kunden gehörte auch der Weimarer Schriftsteller. 
Dennoch ist es lohnend, eine weitere Möglichkeit in Erwägung zu ziehen: Schon seine frühen Studien zeigen, dass Murr ein besonderes Interesse an Byzanz gehabt hat. Es ist bekannt, dass Murrs wissenschaftliche Interessen sich auf viele Gebiete erstreckten, von Philosophie über Mathematik und Naturwissenschaften bis zu Geschichte und Jura. Aber der Nürnberger Universalgelehrte entwickelte mit der Zeit eine besondere Vorliebe für die Altertumswissenschaften und Kunstgeschichte. Erwähnenswert ist dabei seine Dissertation über die diplomatische Geschichte der Stauferkaiser und insbesondere Friedrichs II. (Abb. 2). Unausweichlich war er in diesem Zusammenhang mit der Geschichte und Kultur der Byzantiner konfrontiert, da das schwäbische Kaisergeschlecht eine besondere Beziehung ${ }^{9}$ zur Stadt am Bosporus pflegte. Nach dem Studium und mit diesen Vorkenntnissen begann für Murr eine mehrjährige Phase von Reisen ins Ausland, darunter nach Norditalien und insbesondere Venedig. Dort dürften ihm die vielen „neugriechischen“ Ikonen nicht entgangen sein, und eine davon könnte er dort erworben haben. Dieses Szenario erscheint mir noch wahrscheinlicher, wenn man die Tatsache bedenkt, dass sich in der Lagunenstadt noch heute zwei weitere Ikonen befinden, die aus der gleichen Zeit und aus derselben Werkstatt stammen, wie gleich $\mathrm{zu}$ zeigen ist. Ob Goethe die Ikone Murrs damals noch in dessen Sammlung in Nürnberg bestaunen konnte, und ob die beiden Gelehrten die Gelegenheit dazu hatten, ihre Meinungen zum Thema Ikonenmalerei auszutauschen, bleibt als Gedanke verlockend.

Mit Sicherheit können wir jedoch sagen, dass der Nächste, der eine wissenschaftliche Beurteilung lieferte, wieder aus dem Bekanntenkreis ${ }^{10}$ Goethes stammte. Johann Dominicus Fiorillo kam 1781 an die Universität Göttingen, wo er seine Karriere zunächst als Zeichenlehrer und bald als Dozent für Kunstgeschichte begann. ${ }^{11}$ Hat Fiorillo erst in den letzten Jahrzehnten die Anerkennung in der Wissenschaftsgeschichte bekommen, die ihm gebührt, so hat sein Beitrag zur Erforschung der byzantinischen Kunstgeschichte bisher noch wenig Beachtung ${ }^{12}$ gefunden. Der Mitbegründer der universitären Kunsthistoriographie verstand, wie

9 Zu den bilateralen Beziehungen dieser Mächte siehe zuletzt: K. HEGELE, Die Staufer und Byzanz. Rivalität und Gemeinsamkeit im Europa des Hochmittelalters. Schwäbisch Gmünd 2009, 8- 20. $10 \mathrm{Zu}$ Goethe in Göttingen mit Fiorillo: G. UnverfeHrT, Goethe, Göttingen und die Kunst, in E. Mittler / E. Purpus / G. Schwedt (Hrsg.), „Der gute Kopf leuchtet überall hervor“. Goethe, Göttingen und die Wissenschaft. Göttingen, 99-102.

11 Zuletzt zur Biographie Fiorillos: C. ScHRAPEL, Johann Dominicus Fiorillo. Grundlagen zur wissenschaftlichen Beurteilung der „Geschichte der zeichnenden Künste in Deutschland und den vereinigten Niederlanden“. Hildesheim 2004, 65-81.

$12 \mathrm{Zu}$ den wenigen Ausnahmen gehört: EFFENBERGER, Goethe (wie oben Fußnote 1) 7-23 und 54f. sowie EFFEnBERGER, Ikonenstudium (wie oben Fußnote 1) 255-257. 
wichtig die Göttinger Sammlung in seiner Obhut für die Lehrtätigkeit war, und setzte sie vermehrt ein. Es ist also vorstellbar, dass unsere Ikone auch in diesem Zusammenhang eine Rolle gespielt hat, vor allem bei den Seminaren über die Kunst der Renaissance, die eigentlich der Schwerpunkt der Lehre Fiorillos war.

Als guter Kustos hat er nicht versäumt, die Sammlung in einem Bestandskatalog zu dokumentieren, den er 1805 veröffentlichte, inzwischen als außerordentlicher Professor an der Universität Göttingen. Unsere Ikone wird unter der Nr. 64 vorgestellt und - wie damals für nachantike Objekte üblich - als ,neugriechisch“ bezeichnet. ${ }^{13}$ Zunächst fällt die interessante Bemerkung auf, dass „über diese Arbeit umständlicher geredet wird“. ${ }^{14}$ Daraus ergibt sich einerseits, dass die Ikone Gegenstand von Diskussion gewesen ist, und andererseits, dass sie „umständlicher“ diskutiert wurde, was bezeichnend dafür ist, wie die byzantinische Kunst in dieser Zeit beurteilt wurde. Von Bedeutung ist darüber hinaus der Datierungsvorschlag Fiorillos. Er hielt die Ikone für eine Arbeit des 12. oder 13. Jh.s. Auch wenn die Entstehung dieses Bildwerkes tatsächlich später datiert werden muss, zeigt diese Einschätzung, dass Fiorillo vom wahren Charakter des Bildwerkes etwas richtig erkannt hat. Denn wie noch zu sehen sein wird, gehört der wahre Kern dieser Ikone der Kunst der Palaiologenzeit an, liegt also der von Fiorillo datierten Epoche sehr nahe, was eine für den damaligen Forschungsstand beeindruckende Leistung gewesen ist.

\section{Zwischen Innovation und Tradition: die kretische Koimesis-Ikone in Göttingen}

Auch wenn die kanonischen Evangelien über die letzten Stunden der Gottesmutter auf Erden ganz schweigen, füllen die Apokryphen sowie die Interpretation der Kirchenväter und Kommentatoren des Frühchristentums ${ }^{15}$ diese erzählerische Lücke. Es liegt auf der Hand, dass mit dieser Geschichte wichtige Aspekte einer sehr komplexen theologischen Konzeption verbunden sind: Die Legendenbildung über das Ableben der Mutter Jesu reicht mindestens bis in das 5. Jh. zurück, eine

13 Fiorillo, Beschreibung (wie oben Fußnote 4) 64-65, Kat. Nr. 10.

14 Ebd. 65.

15 Ch. Schaffer, Aufgenommen ist Maria in den Himmel. Vom Heimgang der Gottesmutter in Legende, Theologie und liturgischer Kunst der Frühzeit. Regensburg 1985, 13 - 37. Vgl. auch S.J. SHOEMAKER, Ancient traditions of the Virgin Mary's Dormition and Assumption. Oxford 2003, 977 mit Verweisen auf die ältere Literatur. Vgl. auch S. J. SHOEMAKER, The cult of fashion: the earliest Life of the Virgin and Constantinople 's Marian relics, DOP 62 (2008) 53-74. 
entscheidende Zeit für die Mariologie und die Stellung Mariä im Christentum. ${ }^{16}$ Die Koimesis-Episode beantwortet beispielsweise zugleich Fragen über die Natur Christi, über das Leben nach dem Tode sowie über die Stellung Mariä im Heilswerk Gottes, um nur einige zu erwähnen. All diese dogmatischen, christologischen und mariologischen Ansichten sowie Überlegungen sind die Grundlage für die Genese des Koimesis-Bildes. Das zugehörige Fest wurde von Kaiser Maurikios (582-602) eingeführt. Es wurde am 15. August gefeiert, wie es heute noch in der christlichen Welt üblich ist. Die Koimesis entwickelte sich im Laufe der Zeit zu einem der größten Feste, vielleicht sogar zum zweitwichtigsten Fest der gesamten Christenheit nach Ostern. Ihre Abbildung bildete einen festen und prominenten Bestandteil des Ausstattungsprogramms byzantinischer Monumentalmalerei. ${ }^{17}$ Die Bilderfindung scheint vorikonoklastisch $\mathrm{zu}$ sein, da neben den literarischen Hinweisen über die Existenz dieses Bildthemas vor dem Bildersturm die älteste archäologisch fassbare Darstellung aus der Mitte des 8. Jh.s ausgerechnet in der heutigen Schweiz ${ }^{18}$ erhalten ist. Die Fresken-Fragmente des St.-Martin-Klosters in Disentis legen die Vermutung nahe, dass dieses Festtagsbild bereits in jener Zeit ein festgelegtes Darstellungsschema ${ }^{19}$ besaß, das in dieser Gegend wiederholt

16 Grundlegend zur Mariologie in dieser Zeit: L. Gambero, Mary and the Fathers of the Church. The Blessed Virgin Mary in Patristic thought. San Francisco 1999, 233 - 322. Vgl. auch M. JugIE, La mort et l'assomption de la Sainte Vierge. Étude historico-doctrinale, Vatikan 1944 ; S.C. Mimouni, Les traditions anciennes sur la Dormition et l'Assomption de Marie. Études littéraires, historiques et doctrinales. Leiden 2011.

17 Zur Platzierung und Funktion der Koimesis anhand zweier prominenter Sakralbauten, ChoraKirche in Konstantinopel und Nikolaos-Orphanos-Kirche in Thessaloniki: P. WEIss, Die Mosaiken des Chora-Klosters in Istanbul. Theologie in Bildern aus spätbyzantinischer Zeit. Stuttgart 1997, 164 mit Verweisen auf die ältere Literatur; und K. KIRCHHAINER, Die Bildausstattung der Nikolauskirche in Thessaloniki. Untersuchungen zu Struktur und Programm der Malereien. Weimar 2001, 89-95. Vgl. auch zur Verbindung zwischen Geburt Christi und Koimesis in der byzantinischen Kunst: H. MAgUIRE, Art and eloquence in Byzantium. Princeton 1981, 59-68.

18 Zur Koimesis des St.-Martin-Klosters in Disentis/Mustér im Schweizer Kanton Graubünden: W. STUDER, Disentis/Mustér, St. Martin: Die älteste materiell fassbare Koimesis-Darstellung der Welt. Jahresberichte des Archäologischen Dienstes Graubünden und der Denkmalpflege Graubünden 8 (2004) 64-79, 64-79; sehr gute Abbildungen und Rekonstruktionen sind im Ausstellungskatalog zu finden: W. STUDER, Byzanz in Disentis. Fragmente frühbyzantinischer Monumentalmalerei (Ausstellungskatalog des Rätische Museums Chur vom 18.11.2005 bis 12.03.2006). Chur 2005; zur Disentiser Koimesis und zum gesamten Bildprogramm der Kirche vgl. auch zuletzt W. STUDER, Byzanz in Disentis. Die Reste einer plastisch unterlegten Monumentalmalerei byzantinischer Provenienz des 8. Jahrhunderts aus dem Kloster Disentis. Zürich 2011, 161-210. Überlegungen zur vorikonoklastischen Bilderfindung des Koimesis-Bildes: K. KREIDL-PAPAdoPoulos, Koimesis. RBK 4 (1990) 145-147.

19 Immer noch grundlegend zur Ikonographie des Koimesis-Bildes: G. SCHILLER, Ikonographie der christlichen Kunst 4/2 (Maria). Gütersloh 1980, $92-114$ und Kreidl-PaPadopoulos, Koi- 
wurde. Die ältesten erhaltenen Koimesis-Ikonen stammen hingegen aus nachikonoklastischer Zeit. Sie beinhalten dieselbe ikonographische Grundfassung, indem sie das Ableben der Gottesmutter auf dem Sterbelager im Kreis ihrer Trauergemeinde zeigen.

Die Göttinger Ikone bildet darin keine Ausnahme und folgt treu der etablierten byzantinischen Tradition des Koimesis-Bildes: Hinter der mehrfigurigen Szene auf einem offenen Platz oder Hof ragen zwei Bauten vor goldenem Hintergrund auf. Auf den ersten Blick erscheinen die Bauten mit ihren verzerrt überdehnten Perspektiven eigenartig; sie bestehen aus aufeinander angeordneten kubischen Formen mit Fenstern, Balkonen und mit dunkelfarbenen Tonziegeln auf ihren Pultdächern. Diese Gebäude erfüllen jedoch im Bild zwei wichtige Funktionen: zum einen rahmen sie das ganze Geschehen ein, das sich in der unteren Bildhälfte abgespielt; zum anderen wird der Blick des Betrachters durch die Achsen der Gebäudekanten auf die Hauptpersonen unten im Zentrum des Bildes gelenkt. Farbästhetisch sind darüberhinaus die orangefarbenen Gebäude sehr gelungen, da sie den „flüssigen“ und einwandfreien Übergang vom goldenen Hintergrund zur Menschenmenge im Vordergrund ermöglichen. Hinter den Bauten erscheint jeweils ein Baum, dessen Blätter sehr naturalistisch wiedergegeben ist. Die Bauten könnte man im übrigen als das Haus oder - eine weitere Möglichkeit - als Abbreviaturen der Orte auffassen, wo die abgebildete Episode stattgefunden haben soll. Das Thema der Episode wird oberhalb der Gebäude in zwei Teilen in roten griechischen Majuskeln geschrieben: H KOIMHC[IC] THC $\Theta[$ EOTO]KOY, was so viel bedeutet wie „Das Entschlafen der Gottesmutter“. Wären die beiden Gebäude als Stadt-Abbreviaturen $\mathrm{zu}$ interpretieren, müsste man hier in erster Linie an Bethlehem und Jerusalem denken, dem Bericht der Apokryphen ${ }^{20}$ zufolge die Schauplätze der letzten Stunden Mariä auf Erden.

Die Szene ist fernerhin sehr gut strukturiert; am vorderen Bildrand wird die horizontale Achse durch das große Sterbelager Mariä bestimmt. Die Kline mit dem goldenen Bettgestell ist mit einer schweren purpurroten Decke bedeckt, die mit Vförmig geordneten Falten drapiert ist. Das Betttuch ist durch goldgefärbte Ornamente reich verziert: pseudo-kufische Schriftzeichen, dazwischen Kreise und Rhomben, die schematisierte palmettenähnliche Motive einkreisen, sowie eine breite Bordüre mit ähnlicher Ornamentik wie das Bettgestell. Ergänzt wird die

mesis (wie oben Fußnote 18) 136 - 182; vgl. auch SCHAFFER, Heimgang (wie oben Fußnote 15) 35 -52. Zuletzt auch M. DE GIORGI, Il transito della Vergine. Testi e immagini dall'Oriente al Mezzogiorno medievale. Spoleto 2019, $91-116$.

20 Über die verschiedenen Traditionen zur Lokalisierung des Wohnorts der Maria und der verschiedenen Orte, wo sie ihre letzten Stunden verbracht haben soll, s. zuletzt SHOEMAKER, Ancient traditions (wie oben Fußnote 15) 5-7,103-105, 144. 
prunkvolle Kline durch eine hellrote Matratze und ein olivgrünes Kissen, auf denen der lange, dünne und leblose Körper Mariä ruht. Oberhalb des Kissens erscheint die übliche Beischrift Mariä, die sie als MP $\Theta Y$ (M[HTH]P $\Theta[E O] Y)$, also als Muttergottes bezeichnet. Maria trägt ihre gewöhnliche Kleidung, Tunika und Maphorion, liegt mit geschlossenen Augen und nicht wie in den frühesten Darstellungen mit den Armen verhüllt und eng am Körper angelegt, sondern mit vor der Brust gekreuzten Armen, ${ }^{21}$ wie es vorwiegend in der Kunst der Palaiologenzeit zu sehen ist.Vor der Kline brennen drei Kerzen, wobei der mittlere Kerzenhalter auf einem rechteckigen Podest steht, während die flankierenden Kandelaber über eine dreifüßige Basis verfügen.

Die vertikale Achse wird durch die Darstellung Christi unmissverständlich bestimmt. Christus in einem mit Chrysographie verzierten Gewand erscheint im Zentrum der Komposition hinter der Kline und überragt alle anderen Figuren. Wie Mariä Darstellung mit der Kline besonders betont wird, ist die Darstellung Christi durch die große mantelförmige Aureole besonders hervorgehoben. Die zweizonige Mandorla ist in einer grünfarbenen Grisaille ausgeführt, die von goldenen Lichtstrahlen radial durchbrochen wird. Alles, was von dieser Aureole umschlossen ist, ist mit gleicher Technik gemalt; so die vier Engel mit Kandelabern mit brennenden Kerzen in ihren Händen, jeweils spiegelbildlich zu Zweierreihen übereinander gestaffelt, die Christus flankieren und begleiten; das gleiche gilt auch für den Seraph, der an der Spitze der Aureole über Christus schwebt, wobei sich nur vier seiner Flügelspitzen außerhalb der „Monochromie“ der Aureole befinden, so dass der Unterschied zwischen dem Raum innerhalb und außerhalb der Mandorla noch deutlicher wird. Dieser sechsflügelige Engel gehört ebenfalls zu den ikonographischen Elementen, die in der Palaiologenzeit hinzugefügt wurden. ${ }^{22}$ Man erkennt spätestens jetzt, dass Fioriollo mit seiner Frühdatierung der Göttinger Ikone nicht so falsch lag. Jedenfalls kommt der Seraph zu Details in

21 Auf den frühesten Koimesis-Bildern liegen die Arme Mariä oft eng am Körper an und sind durch ihren Mantel verhüllt, so beispielsweise auf dem Elfenbeinrelief vom Buchdeckel des Evangeliars Kaiser Ottos III. aus der Mitte des 10. Jh.s, das heute in München in der Staatsbibliothek aufbewahrt wird (clm 4453): R. KAHSNITZ, Evangeliar Ottos III.: Elfenbeinrelief mit Koimesis, in R. Baumstark (Hrsg.), Rom und Byzanz. Schatzkammerstücke aus bayerischen Sammlungen [Ausstellungskatalog]. München 1998, 154-158 Kat. Nr. 41. Mit dieser Darstellung des leblosen Körpers Mariä greifen die Künstler auf antike Vorbilder zurück, nämlich auf Totenerweckungs- und Erschaffungsszenen, bei denen tote bzw. seelenlose Körper Bestandteil des Bildes sind; zur Bildformel des Toten vgl. M. Giannoulis, Die Moiren. Tradition und Wandel des Motivs der Schicksalsgöttinnen in der antiken und byzantinischen Kunst. Münster 2010, 62 - 65. 22 Da der Seraph an der Spitze der Aureole zuerst in der Kunst der Hauptstadt überliefert ist, gilt er als Neuerung der Konstantinopler Künstler: KREIdL-Papadopoulos, Koimesis (wie oben Fußnote 18) Sp. $162-164$. 
der Grisaille noch hinzu, die die übernatürliche Lichterscheinung, die unsichtbare himmlische Sphäre bzw. die Theophanie zum Ausdruck bringen. Man sollte nicht vergessen, dass das Koimesis-Bild nicht nur den Tod Mariä, sondern auch die Translation ihrer Seele schildert. Dafür wird ihr eine besondere Ehre erwiesen, indem ihr Sohn persönlich herabkommt, um ihre Seele in Empfang zu nehmen und in den Himmel zu bringen. Somit werden weitere wichtige theologische Sinnschichten des Ereignisses deutlich: die Anwesenden werden gleichzeitig Augenzeugen des Zustandes des auferstandenen Christus, das heißt, der zeitlosen Gottesherrschaft; zugleich ist die Parusie Christi in Verbindung mit der Entrückung der Seele Mariä in den Himmel als Typus des am Ende der Zeit wiederkehrenden Richters aufzufassen. ${ }^{23}$ Die Seele Mariä wird nach antiken Vorbildern als kleines Wickelkind abgebildet. Christus hält sie mit beiden Händen verhüllt, also in einem aus dem antiken Herrscherzeremoniell stammenden Ritus. Ein goldener Nimbus umgibt das Haupt der Seele Mariä, und sie gehört zu den wenigen Figuren neben Christus und der toten Maria, die auch dadurch hervorgehoben werden.

Ein interessantes Detail der Komposition ist darüber hinaus, dass Christus nicht ganz in der Mitte wiedergegeben ist, sondern leicht nach links von der imaginären vertikalen Linie gerückt erscheint, die das Bild in zwei Hälften unterteilt; diese stimmt teilweise mit der zentralen Linie der Gloriole überein, die die Initiale Christi IC XC (I[HCOY]C X[PICTO]C) trennt. Christus hält die Seele Mariä, vom Betrachter gesehen, nach rechts; während sein Körper in angedeutetem Kontrapost steht, wendet er seinen Kopf nach links unten und blickt auf Maria. Mit diesem Kunstgriff ist es dem Maler gelungen, dem Bild Leben und zugleich Gleichgewicht zu verleihen.

Um die beiden Hauptachsen, einerseits die vertikale durch Christus und die Aureole, andererseits die horizontale durch Maria auf ihrem Sterbelager, gruppieren sich die restlichen Figuren der Episode. Auch wenn die Szene über den Tod hinausweist, ist die Thematik vom Alltagesleben bzw. von Klagebildern inspiriert. Es wird der Moment dargestellt, in dem sich Familie und Freunde um das Lager des Verstorbenen versammeln und Abschied nehmen. Die Nachwirkung dieser mindestens bis in die Anfänge der griechischen Kunst zurückreichende Bildtradition ${ }^{24}$

23 Zur theologischen Interpretation der Dormitio in der patristischen Literatur: SCHAFFER, Heimgang (wie oben Fußnote 15) 25-37; vgl. auch SchILlER, Ikonographie (wie oben Fußnote 19) 108.

24 Mit der Darstellung des Sterbelagers und der Trauernden in der Koimesis-Szene wird eine sehr alte Bildtradition fortgeführt. Diese begegnet bereits in der Kunst der geometrischen Zeit, etwa auf der bekannten Amphore des Athenischen Friedhofs am Dipylon - um nur ein Beispiel zu nennen mit der Szene einer Aufbahrung samt der Trauergemeinde; zur Amphore, aber auch zur Mimik und 
prägt die Ikonographie auch dieser Koimesis-Ikone ganz deutlich. Der emotionale Zustand all dieser Personen wird dem Betrachter durch ein elaboriertes System an Gesten und Körperhaltungen vermittelt. ${ }^{25}$ Alle Figuren stehen mit gebeugtem Haupt, manche davon legen die Hand an die Wange oder greifen an den Bart, während einige andere mit dem Bausch ihrer Gewänder ihre Gesichter zu verbergen suchen; auf diese Weise wird ihre tiefe, jedoch zugleich zurückhaltende Trauer zum Ausdruck gebracht. Die Trauergemeinde Mariä setzt sich, der Legende und der Bildtradition der palaiologischen Epoche folgend, oben rechts aus Frauen, den Begleiterinnen der Gottesmutter, aus den Aposteln, die wundersamerweise nach einer Wolkenreise aus allen Ecken der Welt erscheinen, und aus den drei Hierarchen zusammen.

Die Bischöfe treten durch ihre Omophoria aus der Menschenmenge hervor. Links befindet sich Jakobus, der sogenannte Herrenbruder, Bischof von Jerusalem. Rechts sind Dionysios Areopagites sowie Timotheos von Ephesos - der jüngste von den insgesamt dreien - mit einem Weihrauchgefäß bzw. mit einem Katsion in der rechten Hand zu sehen. Die beiden älteren Bischöfe halten mit beiden Händen verhüllt je einen aufgeschlagenen Codex, auf dem griechische Buchstaben geschrieben sind. Die Fläche der offenen Codices war dem Maler anscheinend zu klein, um einen Text zu schreiben, wie es oft bei anderen Koimesis-Ikonen der Fall ist. Jedoch ist links vom Betrachter, auf dem Codex von Jakobus, bei näherer Betrachtung deutlich das erste Wort zu erkennen; hier steht das Wort „H Z $\Omega \mathrm{NH}^{\circ}$, was „der Gürtel“ bedeutet. ${ }^{26}$ Das Wort ist orthographisch richtig sowie mit der richtigen Akzentuierung geschrieben. Weitere Indizien für das Bildungsniveau des Malers $^{27}$ der Ikone, der offenbar gut lesen und schreiben konnte, sind die richtige Form aller Buchstaben und die versierte Verwendung von Ligaturen. Diese verraten ebenfalls eine erfahrene Hand. Die Buchstaben sind für das bloße Auge zu

Gestik in der antiken Kunst im Zusammenhang mit Tod und Trauer s. zuletzt mit älterer Bibliographie: I. HubER, Die Ikonographie der Trauer in der griechischen Kunst. Mannheim 2001, 67 70.

25 Die Vorbilder der byzantinischen Künstler und die Darstellung der Trauergestik und -mimik in der frühchristlichen und byzantinischen Kunst hat Henry Maguire in einem sehr informativen und ausführlichen Artikel behandelt; wenn dieser in der Forschung nicht die Beachtung erfuhr, die er verdient hätte, so vielleicht aus dem Grund, dass er ein wenig seiner Zeit voraus war: H. MAGUIRE, The depiction of sorrow in middle Byzantine art. DOP 31 (1977) 123-174.

26 Ich möchte mich an dieser Stelle bei Manolis Patedakis und Andreas Rhoby für ihre Hilfe bei der Entzifferung der Schrift auf den Codices bedanken.

27 Die Grundausbildung in Lesen und Schreiben für die jungen Maler gehörte in der Regel zu den Aufgaben des Meisters; dazu und zum Bildungsniveau der kretischen Maler mit weiterführender

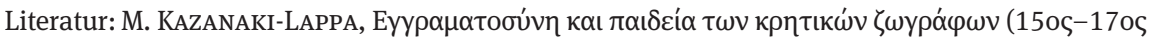

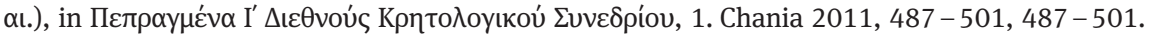


klein. Erst durch eine sehr nahe und sehr aufmerksame Betrachtung erkennt man, dass der mikrographische Text griechische Codices imitiert. Daraus kann der Schluss gezogen werden, dass der Maler über eine Linse verfügte, sodass er durch die optische Vergrößerung in der Lage war, die winzigen Buchstaben mit dieser Präzision auszuführen.

Das Wort des Codex „der Gürtel“ bezieht sich auf die legendäre Gürtelspende an Thomas, der viel später als die anderen Apostel vor Ort erschien; Thomas konnte nur die Himmelfahrt Mariä erleben, wobei er ihren Gürtel erhielt. Durch die Hinzufügung des Wortes auf dem Codex ist dem Maler eine besonders interessante Variante einer Text-Bild-Interaktion gelungen, die auf das Marienbild und die Marienverehrung verweist. Die Darstellung der Aufnahme Mariä in den Himmel mit der Gürtelspende ${ }^{28}$ an Thomas gehört zu den häufigsten bzw. kanonischen Nebenszenen des Koimesis-Bildes. Die Darstellung der Göttinger Ikone konzentriert sich auf das Hauptereignis der Koimesis, jedoch wollte der Maler nicht ganz auf die Bedeutung der Gürtelepisode verzichten. Der Gürtel Mariä29, der in byzantinischer Zeit eine der wichtigsten Marienreliquien Konstantinopels war, gehörte nach wie vor zu den Gegenständen, die immer wieder durch zahlreiche Wunder ihre „Echtheit“ bewiesen, und war zugleich der sichtbare Beweis für die Hauptereignisse um den Tod Mariä. Auf jeden Fall ist es hier wichtig zu bemerken, dass dem Maler die besondere Bedeutung die Gürtellegende für das Koimesis-Bild bewusst war; mit der Erwähnung des Gürtels wird der Gegenstand in den Vordergrund gerückt, der gleichzeitig Metapher und Zeichen für die Jungfräulichkeit, die Stellung Mariä als Gottesgebärerin und ihre Assumptio ist. Dem Maler gelingt es hier, wie einem Dichter, durch ein einziges Wort auf dem Codex die Hauptaspekte des Koimesis-Bildes „zusammenzufassen“.

Die beiden im Hintergrund gestaffelten Figuren-Gruppen werden jeweils von einem Apostel angeführt. Anhand ihrer physiognomischen Merkmale, die schon in der frühchristlichen Zeit standarisiert sind, sind die Apostelfürsten leicht zu erkennen. Mit etwas breiterem Kopf, weißen, bis in die Stirn reichenden Haaren und rundem Bart erscheint Petrus, der ein Weihrauchgefäß schwenkt. Apostel Paulus rechts, mit dunkleren Haaren, Vorderglatze und spitzem Bart, mit tief gebeugtem Oberkörper, berührt die Füße Mariä. Auch wenn die Haltung und die Geste des Paulus der Situation angepasst sind, sind sie wiederum unverkennbar

28 SCHILlER, Ikonographie (wie oben Fußnote 19) 119-123.

29 Zum Mariengürtel und seiner Bedeutung sowie seiner Geschichte und seinen Vorbildern s. mit Verweis auf die ältere Literatur C. Sсноррноғғ, Der Gürtel: Funktion und Symbolik eines Kleidungsstücks in Antike und Mittelalter. Köln 2009, 215 - 220. SHOEMAKER, Ancient traditions (wie oben Fußnote 15) 61-66. 
dem aus dem antiken Herrscherzeremoniell ${ }^{30}$ stammenden Ritus entlehnt und auf Maria übertragen. Es ist m. E. davon auszugehen, dass mit der Körperhaltung der trauenden und gleichermaßen huldigenden Apostel die Verehrung Mariä zum Ausdruck gebracht wird. Auch wenn der Trauercharakter, wie bereits erwähnt, des Geschehens durch ihre gebeugte Körperhaltung thematisiert wird, ist diese zugleich einem ähnlichen Bildtypus wie die Proskynese von Paulus verpflichtet; je näher vom Sterbelager sich die Figuren demgemäß befinden, desto nachdrücklicher wird ihre adoratio vollzogen. So befindet sich beispielweise neben Paulus auch Timotheus in tief gebeugter Körperhaltung und neben ihm die Figur, die aufgrund der typischen Porträtzüge mit Johannes dem Theologen zu identifizieren ist.

Solche physiognomischen Merkmale, durch die sich auch die anderen Apostel auf dem Bild unschwer erkennen lassen, bieten zudem auch den wichtigsten Anhaltspukt für die Datierung der Göttinger Ikone. Die Apostelgesichter der Göttinger Ikone weisen genau die Merkmale auf, die sich in der frühen sogenannten „kretischen“ Malerei entwickelt haben. Nicht nur auf Bildern mit der gleichen Thematik des 15. Jh.s, sondern auch auf den zeitgleichen ChristusWeinstock-(Ampelos)-Ikonen sind die gleichen Apostelgesichter deutlich zu erkennen. ${ }^{31}$ Die Vergleichsbeispiele führen uns auf die Ikonenmalerei Kretas um das 15. Jh. Aus dieser Zeit stammt eine Reihe von kretischen Koimesis-Ikonen, die sowohl stilistisch als auch ikonographisch mit der Göttinger-Ikone verwandt sind. Hierzu gehören, um nur einige zu nennen, die Koimesis-Ikonen des Benaki-Museums, ${ }^{32}$ der Latsis-Privatsammlung ${ }^{33}$ oder des Aikaterini-Klosters in Candia (Heraklion); letztere wird heute in Zakynthos aufbewahrt. ${ }^{34}$

30 Zur Bedeutung der Proskynese und der Geste, die Füße zu berühren: J.G. DEcKERs, Gnade für Hades? Beobachtungen am Bild der Anastasis. JbAC 50 (2007) 130-138.

31 Zur Genese und Bedeutung des Ampelos-Bildes: A. ManTAS, The iconographical subject „Christ the Vine“ in Byzantine and post-Byzantine art. DChAE 24 (2003) 347 -360. Vgl. auch die drei Ampelos-Ikonen auf denen die Porträts der Apostel samt Beischriften deutlich zu erkennen sind: M. Borboudakis, Angelos, Christ the Vine, in M. Vasilaki (ed.), Xeip 'Ayyćરou. The hand of Angelos. An icon painter in Venetian Crete (Ausstellungskatalog, 16. November 2010 - 31. Januar 2011, Benaki Museum). Athen 2010, 158 - 163, Kat. Nr. 28 - 30. Zu Gesichtszügen der Personen

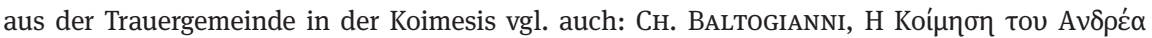

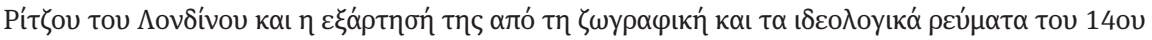

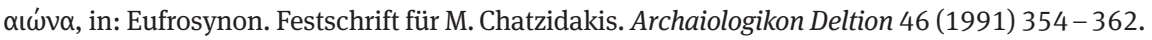

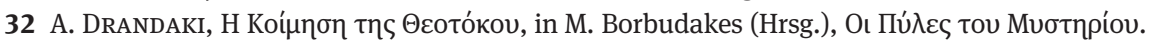

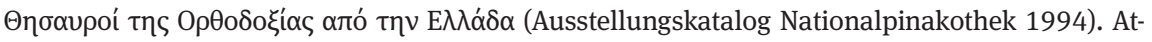
hen 1994, 238-239 Kat. Nr. 59.

33 V. Fotopoulos / S. Runciman, Meta to Byzantio. The survival of Byzantine sacred art (Ausstellungskatalog, Hellenic Centre London 1996). London 1996, $30-31$, Kat. Nr. 4, mit älterer 
Viel näher jedoch steht die Göttinger Ikone einer weiteren Gruppe von Koimesis-Ikonen, die alle dem Umkreis des in Candia ansässigen kretischen Malers Andreas Ritzos zugeschrieben werden. Mit der Göttinger Ikone, so scheint es, schließt sich der Kreis dieser Koimesis-Ikonen. In seinem Zentrum befindet sich die einzige Koimesis-Ikone, an deren Autorschaft nicht gezweifelt werden kann, da sie vom Meister selbst signiert ist. Diese Tafel (Abb. 3), die heute in der Galleria Sabauda in Turin ausgestellt wird, stellt innerhalb der Gruppe die bemerkenswerteste und qualitativ hochwertigste Arbeit dar. Sie trägt also nicht ohne Grund allein das Autogramm des Meisters, ${ }^{35}$ der in Candia einem berühmten Atelier vorstand. Beim Vergleich zwischen der Ikone des berühmtesten kretischen Malers aus der zweiten Hälfte des 15. Jh.s mit der Göttinger sind die stilistische und ikonographische Verwandtschaft der beiden Bildwerke unverkennbar: Das wird zunächst durch die ähnliche Architekturkulisse und die „durchsichtigen“ monochromen Engel besonders deutlich. Die enge Beziehung zwischen dem (Vor-) Bild des Meisters und der Göttinger Ikone wird auch bei Bildelementen von sekundärer Bedeutung besonders klar. Dazu zählen unter anderem die identische Ornamentik der Goldbordüre des Betttuches, die pseudo-kufischen Schriftzeichen, die schrägen Kannelierungen der Kandelaber vor der Kline sowie die gleichförmigen Kissenecken unter dem Kopf Mariä (Abb. 4). Es ist bekannt, dass die Meister solche Details oft von ihren Gehilfen ausführen ließen. Daher kann m. E. davon ausgegangen werden, dass der Schüler des kretischen Ateliers, der für die sekundären Motive der Turiner Ikone zuständig war, auch die Göttinger Ikone angefertigt hat.

Der Vollständigkeit halber sei erwähnt, dass auch einige Unterscheide der beiden Werke auffallen. So nimmt bei der Turiner Ikone eine viel größere Zahl an Personen am Hauptgeschehen teil als bei der Göttinger Ikone. Allein die Seite zur Rechten Christi wird fast ausschließlich von der Schar der Engel eingenommen. Auch das Sterbelager Mariä, jetzt platziert von rechts nach links, verleiht durch seine Schrägstellung der ganzen Komposition eine neue „Verräumlichung“. Ebenso verraten die Art und Weise, wie das Gesicht Christi modelliert ist, mit seinen milden Gesichtszügen und mit der feinen Ausführung der Bart- und Haartracht die erfahrene Hand des Meisters. Der Grund für diese Unterschiede ist,

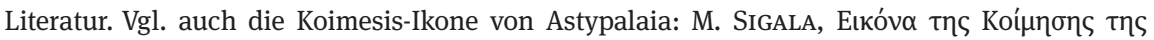

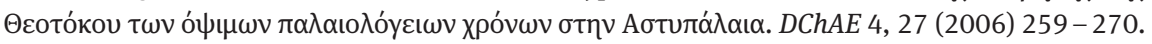

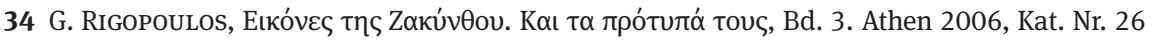
Abb. $298-300$.

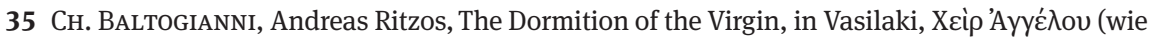
oben Fußnote 31) Kat. Nr. 50, 202 - 204 mit älterer Literatur. 
dass es sich bei der Turiner Ikone um ein Meisterwerk bzw. um eine besondere Auftragsarbeit handelt, die Andreas Ritzos selbst signierte.

Die anderen Ikonen dieser gemeinsamen Gruppe, oder besser gesagt die „Schwester“-Ikonen der Göttinger Koimesis sind: 1. Das Triptychon mit der Koimesis-Ikone (Abb. 5), dem Hl. Dominicus und Hl. Franciscus, heute im PuschkinMuseum, ${ }^{36}$ 2. und 3. die beiden Koimesis-Ikonen (Abb. 6) des Instituto Ellenico ${ }^{37}$ in Venedig sowie 4. die Koimesis-Ikone des Iviron-Klosters ${ }^{38}$. $\mathrm{Zu}$ diesen muss auch die Deesis-Ikone des Sohnes von Andreas Ritzos, Nikolaos in Sarajevo hinzugefügt werden $^{39}$; die letzte Szene der kleinen Rahmendarstellungen ist wiederum eine sehr ähnliche Koimesis. Stellt man alle diese Ikonen nebeneinander, ist die Ähnlichkeit so verblüffend, dass kein Zweifel mehr daran bestehen kann, dass diese aus der gleichen Werkstatt stammen und auf das Ende des 15. Jh.s datiert werden müssen. Als erstes fällt sicherlich die identische Verteilung der Figuren in der Fläche der Tafel auf (Abb. 7): Um nur ein besonders deutliches Beispiel herauszugreifen, wird Simon Zelotes stets rechts oben in die Szene gesetzt, der einzige Apostel dort unter dem rechten Gebäude mit der charakteristischen Vorderglatze mit weißem, kurzem und rundem Bart (Abb. 8). Die ähnlich mandelförmige Aureole und die jeweils zwei Engel links und rechts in Grisaille sind ebenfalls in allen diesen Bildern vorhanden. Auch die leichte Verschiebung der in Kontrapost stehenden Figur Christi von der zentralen vertikalen Achse ist fast auf allen diesen Bildern zu sehen. Malweise, Kolorit und Ornamentik der Kline Mariä weisen weitere erstaunliche Ähnlichkeiten auf; bei diesen Klinen sind u.a. die stilisierten Palmetten und die sogenannten pseudo-kufischen Schriftzeichen zu vergleichen, die oft in der kretischen Ikonenmalerei anzutreffen sind. Die Liste der Gemeinsamkeiten dieser Ikonen könnte beliebig erweitert werden, von der Tendenz zur Schlankheit der Figur Mariä bis zur identischen Körperhaltung des Paulus in Proskynese, der immer wieder in dunkelgrünen Chiton und dunkelrotes Himation gekleidet zu Füßen der Muttergottes erscheint (Abb. 8).

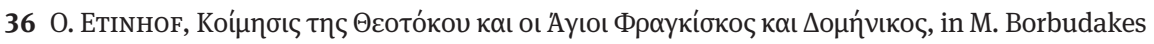

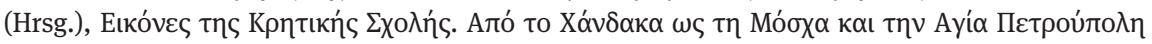
(Ausstellungskatalog 15. September-30. Oktober 2011, Basilika S. Marco / Ag. Aikaterini-Kloster in Heraklion). Heraklion 2004, 438 - 440, Kat. Nr. 87 mit älterer Literatur.

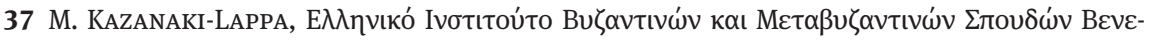

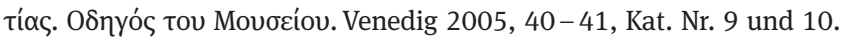

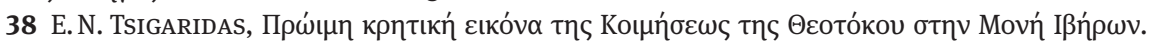
DChAE 28 (2007) 193-203, Abb. 1.

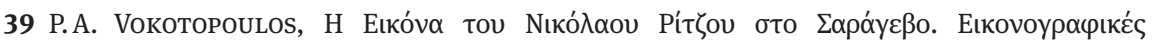

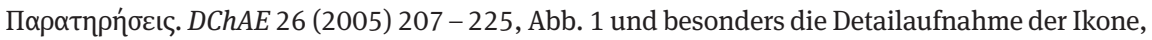
Abb. 11. 
Allerdings muss betont werden, dass keine dieser Ikonen mit einer anderen vollkommen identisch ist. Trotz standardisierter Werkformen, beispielsweise mit Anthivola, wissen die Maler in Ritzos' Atelier ihre Arbeit zu variieren und originell zu sein; die bewusste Mimesis der palaiologischen Tradition bedeutet in keinem Falle eine sklavische Kopie oder mechanische Reproduktion des Themas. So werden Komplementärszenen hinzugefügt oder nicht, z. B. die Wolkenreise der Apostel wird auf beiden Ikonen des Instituto Ellenico gemalt (Abb. 6), jedoch nur auf der ersten von beiden wird die Gürtelspende an Thomas und die Aufnahme Mariä in den Himmel wiedergegeben. Auch zwischen der Göttinger Ikone (Abb. 1), der zweiten Ikone des Instituto Ellenico (Abb. 6) und der Ikone vom Puschkin Museum (Abb. 5), die sich innerhalb dieser Gruppe am nächsten stehen, sind ikonographische und stilistische Unterschiede $\mathrm{zu}$ erkennen. So wird den rahmenden Gebäuden auf der Puschkin-Museum-Ikone statt den Pultdächern in den beiden Ikonen anderen hier ein Ziborium als Dachabschluss hinzugefügt. Auf der Ikone im Puschkin-Museum und der zweiten Ikone des Instituto Ellenico schwebt kein Seraph an der Spitze der Aureole, wie es auf der Göttinger Ikone der Fall ist. Solche Variationen und Modifikationen einer seit Jahrhunderten tradierten Thematik sind nicht nur auf bestimmte Wünsche der verschiedenen Auftraggeber, sondern auf die feinsinnige künstlerische Inspiration der kretischen Maler zurückzuführen; es fällt ihnen leicht, die byzantinische Tradition zu bewahren und zugleich originell $\mathrm{zu}$ sein. ${ }^{40}$

\section{Die „Auferstehung“ der Göttinger Koimesis-Ikone in der postbyzantinischen Epoche}

Die Ikonen aus dem Atelier des Ritzos wurden von der Nachwelt als etwas Besonderes empfunden. Das beweist vor allem die Göttinger Ikone, die als Vorbild für die Maler des 17. Jh.s diente. Von den vielen Beispielen sind zwei Werke zu erwähnen: die Koimesis-Ikone des Malers Emmanuel Lambardos um 1600, die heute auf Korfu aufbewahrt wird, ${ }^{41}$ und die Koimesis-Ikone des Malers Viktor,

40 Vgl. auch M. ConstantoudaKi-Kitromelides, Tradition and diversity: icon cainting in Crete, Venice and the Ionian Islands and El Greco's early career, in P. Kitromilides / D. Arvanitakis (Hrsg.), The Greek world under Ottoman and Western domination: 15th-19th centuries (Proceedings of the International Conference. A.S. Onassis Foundation, 29 April 2006 New York). New York / Athen 2008, 55-79.

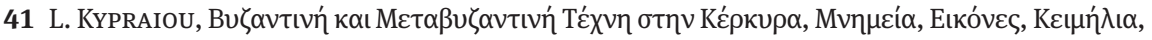

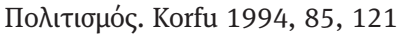


heute im Instituto Ellenico in Venedig, ${ }^{42}$ aus der zweiten Hälfte des 17. Jh.s. (Abb. 9). Hier geht wiederum die Ähnlichkeit mit den kretischen Vorbildern des 15. Jh.s so weit, dass man Schwierigkeiten hätte, sie richtig zu datieren, wenn beide nicht signiert wären. Besonders Viktors Ikone stimmt in allen Einzelheiten mit der Göttinger Ikone überein, was die Annahme zulässt, dass gerade sie Viktor als unmittelbares Vorbild diente. Abgesehen von Nebenszenen - die Episode des Iephonias im Vordergrund und die Wolkenreise der Apostel sowie die Aufnahme Mariä in den Himmel im Hintergrund - sind alle anderen Einzelheiten auf der Ikone Viktors detailgetreu übernommen. Gestik und Körperhaltung jeder einzelnen Figur auf Viktors Ikone stimmt genau mit der Göttinger Ikone überein. Das gleiche gilt auch für die Gebäude im Hintergrund, die identisch sind, lediglich in grauer Farbe.

Bei aller Ähnlichkeit mit dem Vorbild fallen die Eigenheiten des Malers Viktor und der späteren Epoche bei genauerer Betrachtung durchaus ins Auge: Der aufmerksame Betrachter erkennt, dass der Faltenwurf in Viktors Ikone anders ist als der auf der Göttinger Ikone. Viktor zeigt eine Vorliebe für gröbere tiefere schwarze Y-förmigen Falten, wie sie z. B. auf dem Himation Petri gut zu erkennen sind (Abb. 10). Auch die Modellierung des Inkarnats ist bei Viktors Figuren anders, so beim Gesicht Christi oder Mariä, mit dunkleren Schattierungen und intensiverer Verwendung der schwarzen Farbe. Tatsächlich ist die Episode bekannt, bei der ein Priester aus Venedig und ehemaliger Kunde sich über die Qualität einer Ikone Viktors beschwerte. ${ }^{43}$ Entgegen seinen Erwartungen waren dem Priester u.a. das Gesicht der Muttergottes nicht schwarz bzw. dunkel genug. Anscheinend gehörten die dunkleren Fleischfarben der Figuren zu den stilistischen Vorlieben des 17. Jh.s, die bei diesem Koimesis-Bild auch zum Vorschein kommen. Die Tatsache, dass die Göttinger Ikone Pate gestanden haben muss, ist jedoch m.E. kein Beweis für mangelnde Inspiration der Maler des 17. Jh.s. Es ist vielmehr eine Bestätigung, welche hohe Wertschätzung die späteren Generationen dieser Bilderwerke der frühen kretischen Schule zukommen ließen.

Dass Viktor nach unserem jetzigen Wissen ${ }^{44}$ die meisten Jahre seines Lebens in Candia (Heraklion) verbrachte, führt uns zu dem Schluss, dass sich die Göttinger Ikone in der zweiten Hälfte des 17. Jh.s wahrscheinlich noch dort befand. Die Koimesis von Viktor und die beiden anderen Koimesis-Ikonen aus dem Atelier von

42 KaZANAKI-LAPPA (wie oben Fußnote 37), 79 Kat. Nr. 42.

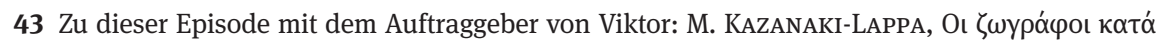

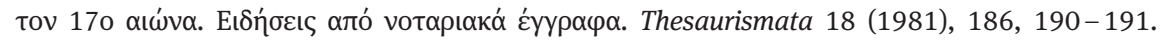

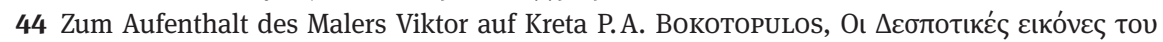

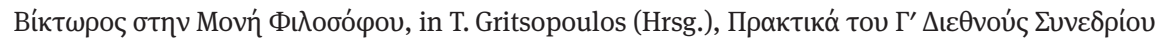

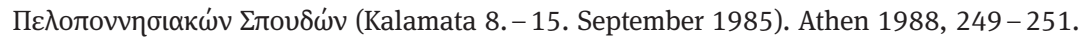


Ritzos tauchen dann in Venedig auf, wo sie sich bis heute befinden. Es ist daher nicht abwegig anzunehmen, dass die Göttinger Ikone aus dem Atelier von Ritzos zusammen mit den anderen Ikonen nach Venedig gelangte. Dort hatte wahrscheinlich Gottlieb von Murr die Gelegenheit, die Göttinger Ikone zu erwerben.Von dort gelangte sie nach Deutschland, wo sie ihren Beitrag zum besseren Verständnis der kretischen Ikonenmalerei leisten konnte. 